\title{
DESIGN-ORIENTED ANALYSIS OF AIRCRAFT FUSELAGE STRUCTURES
}

\author{
Gary L. Giles* \\ NASA Langley Research Center, Hampton, Virginia
}

\begin{abstract}
$\underline{\text { Abstract }}$
A design-oriented analysis capability for aircraft fuselage structures that utilizes equivalent plate methodology is described. This new capability is implemented as an addition to the existing wing analysis procedure in the Equivalent Laminated Plate Solution (ELAPS) computer code. The wing and fuselage analyses are combined to model entire airframes.

The paper focuses on the fuselage model definition, the associated analytical formulation and the approach used to couple the wing and fuselage analyses. The modeling approach used to minimize the amount of preparation of input data by the user and to facilitate the making of design changes is described. The fuselage analysis is based on ring and shell equations but the procedure is formulated to be analogous to that used for plates in order to take advantage of the existing code in ELAPS. Connector springs are used to couple the wing and fuselage models.

Typical fuselage analysis results are presented for two analytical models. Results for a ring-stiffened cylinder model are compared with results from conventional finite-element analyses to assess the accuracy of this new analysis capability. The connection of plate and ring segments is demonstrated using a second model that is representative of the wing structure for a channel-wing aircraft configuration.
\end{abstract}

$$
\begin{array}{ll} 
& \text { Nomenclature } \\
b, h, z \quad= & \text { dimensions of rectangular portion of ring } \\
& \text { cross-section [see Fig. 3] } \\
E & =\text { modulus of elasticity } \\
& =\text { spring stiffness } \\
k_{s} & =\text { lamina stiffness matrix } \\
Q & =\text { radius of shell reference surface } \\
r & =\text { circumferential directions } \\
R_{l}, R_{2} \quad= & \text { radii of curvature in meridional and } \\
s & =\text { meridional shell coordinate } \\
t & =\text { thickness of cover skin layer } \\
u, v, w & =\text { displacements in Cartesian coordinates } \\
\bar{u}, \bar{v}, \bar{w} & =\text { displacements in shell coordinates } \\
x, y, z & =\text { Cartesian coordinates } \\
\delta_{s e g} & =\text { deflection of segment at spring connection } \\
\varepsilon_{s} & =\text { strain in meridional direction } \\
\varepsilon_{\theta} & =\text { strain in circumferential direction } \\
\varepsilon_{s \theta} & =\text { shear strain }
\end{array}
$$

\footnotetext{
* Senior Aerospace Engineer, Systems Analysis Branch, Aeronautics Systems Analysis Division, Airframe Systems Program Office. Associate Fellow AIAA.

Copyright (C) 1998 by the American Institute of Aeronautics and Astronautics, Inc. No copyright is asserted in the United States under Title 17, U.S. Code. The U.S. Government has a royalty-free license to exercise all rights under the copyright claimed herein for Governmental Purposes. All other rights are reserved by the copyright owner.
}

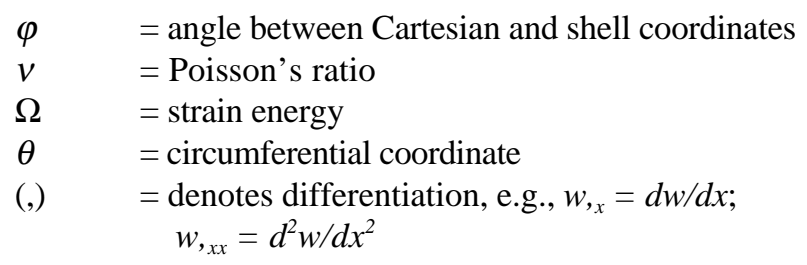

\section{Introduction}

During the conceptual design of aircraft, many alternative configurations must be evaluated in multidisciplinary design trades to determine the characteristics of a candidate configuration which will best meet specified measures of overall vehicle performance and/or cost. Airframe weight is the key parameter that is required from the structures discipline. The airframe should be lightweight but also have sufficient strength and stiffness necessary to satisfy all the requirements throughout the flight envelope. General-purpose finite-element structural-analysis codes are available to model and analyze the static and dynamic response of airframes in great detail. However, such analyses often require several months to generate the finite-element model and repetitive analyses can be computationally expensive. With the objective of 
reducing design-cycle time, equivalent plate methodology ${ }^{1-4}$ has been developed and used for analysis of aircraft wings and empennage structures during early phases of design. In order to model entire airframes, similar capabilities are needed for modeling other structural components.

This paper describes a design-oriented analysis capability for aircraft fuselage structures. This new capability has been implemented as an addition to the existing wing analysis procedure in the Equivalent Laminated Plate Solution (ELAPS) computer code $^{5}$. Thus, the wing and fuselage analyses are now combined so that entire airframes can be modeled.

The paper focuses on the fuselage model definition, the associated analytical formulation and the approach used to couple the wing and fuselage analyses. The modeling approach used to minimize the amount of preparation of input data by the user and to facilitate the making of design changes is described. The fuselage analysis is based on ring and shell equations but the procedure is formulated to be analogous to that used for plates in order to take advantage of the existing code in ELAPS. Connector springs are used to couple the wing and fuselage models.

Typical fuselage analysis results are presented for two analytical models. Results for a ring-stiffened cylinder model are compared with results from conventional finite-element analyses to assess the accuracy of this new analysis capability. The connection of plate and ring segments is demonstrated using a second model that is representative of the wing structure for a channelwing aircraft configuration.

\section{Analytical Modeling}

Fuselage structures are modeled as ring-stiffened shell segments, typical of transport aircraft construction, as shown in Fig. 1. Each shell segment is used to represent large regions of a fuselage and only a small number of segments are typically used to model an entire fuselage. These segments have circular cross sections with a radius that varies along the length to represent area ruling and the necking down in the fore and aft regions of a typical vehicle. The skin of the shell segments are composed of layers of orthotropic material. The properties of the layers can be defined to represent composite laminates or a smeared representation of longerons. Fuselage frames are modeled with rings having cross sections that are composed of multiple rectangles.
The shell segments are defined over quadrilateral regions with boundary edges at constant values of $x$ along the length and at constant values of $\theta$ in the circumferential direction as shown in Fig. 2a. Segments for half airplane models, that are symmetric about the $x-z$ plane, are often defined with $\theta$ extending from $-\pi / 2$ to $+\pi / 2$. An appropriate set of boundary conditions must be applied at $\theta=-\pi / 2$ and at $\theta=+\pi / 2$. Small segments with a negative thickness can be superimposed on these larger segments to represent cutouts such as doors or windows. The radius of the reference surface for the shell is defined by a polynomial function along the length of the segment.

$r(x)=r_{0}+r_{1} x+r_{2} x^{2}+r_{3} x^{3}+\mathrm{L}+r_{n} x^{n}$

The skin of the shell segments consist of orthotropic layers with the thickness of each layer being defined independently by a two-dimensional polynomial function along the length and around the circumference.

$t_{k}(x, \theta)=t_{00}+t_{10} x+t_{20} x^{2}+t_{01} \theta+\mathrm{L}+t_{m n} x^{m} \theta^{n}$

Orientation of the stiffness properties and corresponding thickness are specified for each layer, and the orientations and thicknesses can be different in different shell segments.

Fuselage frames are modeled with rings having cross sections that are defined using up to three rectangles so that a variety of shapes (e.g., zee, tee, cee) can be represented. An example of a zee cross section is shown in figure 3 that indicates the width, $b$, height, $h$, and the distance of the centroid from the shell reference surface, $z$, must be defined for each rectangle in the cross section. The dimensions of cross sections at each end of a shell segment must be defined along with the total number of frames, $N$, to be equally spaced along the length of the segment. At frames between the ends, the cross sectional dimensions are interpolated using a linear variation between dimensions of rectangles that are defined at the ends of a shell segment. During the analysis, the bending and extensional stiffnesses of these frames are smeared over the surface of the shell. This smeared approximation provides improved computational efficiency. Individual, discrete frames can be modeled using additional narrow shell segments with a width equal to the width of the discrete frame shell. The behavior of discrete frames will be closely approximated when the frame stiffnesses are smeared over these narrow segments. The option of using discrete or smeared modeling can be used to trade 
accuracy for computational speed, a desirable feature of design-oriented analysis methods.

\section{Analysis Procedure}

The analysis procedure is based on the Ritz method in which the deflection of the structure is described by assumed polynomial displacement functions. Axial, tangential and radial $(u, v, w)$ deflections are defined in terms of functions in the axial, $x$, and circumferential, $\theta$, coordinates on the surface of a shell segment as shown in Fig. 2b. Note that the radial deflection is defined to be normal to the $x$-axis rather than the conventional definition of being normal to the shell surface. This definition of radial deflection facilitates the coupling of shell segments to plate segments. The assumed displacement functions are substituted into the expression for total energy. This equation is differentiated with respect to each of the displacement function coefficients to minimize the total energy. A set of linear, simultaneous equations is produced that can be solved for the desired set of unknown polynomial coefficients. These coefficients are used to calculate deflections, strains and stresses at a user specified grid of points over the surface of the shell segments.

The total energy consists of the strain energy of the structure and the virtual work associated with the applied loads and assumed displacement functions. The strain energy of the structure has contributions from the shell segments and the frames. Only the membrane energy of the shell is used while energies associated with both extension and bending are included for the ring frame. The strain equations are derived from ring and shell theory.

\section{Strain Energy of Shell Segments}

The membrane strain energy of a shell segment is given as

$$
\Omega_{\text {shell }}=1 / 2 \iint_{\text {area }}\{\varepsilon\}^{T}[Q]\{\varepsilon\} d A
$$

where

$$
\{\varepsilon\}^{T}=\left\{\begin{array}{lll}
\varepsilon_{s} & \varepsilon_{\theta} & \varepsilon_{s \theta}
\end{array}\right\}
$$

Strain-displacement relations and the differential area in terms of conventional shell coordinates are given in Ref. 6 as

$$
\begin{aligned}
& \varepsilon_{s}=\bar{u},{ }_{s}+\bar{w} / R_{1} \\
& \varepsilon_{\theta}=\bar{v},{ }_{\theta} / r+\bar{u} r,{ }_{s} / r+\bar{w} / R_{2} \\
& \varepsilon_{s \theta}=\bar{u},_{\theta} / r+\bar{v},{ }_{s}-\bar{v} r,{ }_{s} / r
\end{aligned}
$$

and $\quad d A=r d \theta d s$

where $\theta$ is the circumferential coordinate and $s$ is the meridional coordinate. The displacement $\bar{w}$ is normal to the shell reference surface and the displacements $\bar{u}$ and $\bar{v}$ are in the meridional and circumferential directions respectively. In order to make the fuselage shell formulation be analogous to the plate formulation for wings, quantities in these strain-displacement equations are transformed to the Cartesian coordinate system shown in Fig. 2b. Formulating energy expression in this form, facilitates the coupling of the shell and plate segments. The quantities in the expression for strain energy after transformation are

$$
\begin{aligned}
& \varepsilon_{s}=\left(u,{ }_{x}+r,{ }_{x} w,{ }_{x}\right) /\left(s,{ }_{x}\right)^{2} \\
& \varepsilon_{\theta}=\left(v,,_{\theta}+w\right) / r \\
& \varepsilon_{s \theta}=\left(u,{ }_{\theta}-r,{ }_{x} v+r v,{ }_{x}+r,{ }_{x} w,{ }_{\theta}\right) /\left(r s,{ }_{x}\right)
\end{aligned}
$$

and

$$
d A=r s,{ }_{x} d \theta d x
$$

where $\quad s,{ }_{x}=\sqrt{1+r,{ }_{x}^{2}}$

The details of the transformation procedure are given in Appendix A.

\section{Strain Energy of Ring Frames}

Only in-plane displacements of the ring frames are included in this formulation; out-of-plane bending and torsion are neglected. The general equations for the strain energy of a ring are given in Ref. 6 in terms of the same Cartesian coordinate system that is used for the shell in the preceding subsection. Therefore, the inplane contributions are taken directly from these equations and are written in the nomenclature of this paper as

$$
\Omega_{\text {ring }}=1 / 2 \int_{\theta} \int_{A}\left\{\varepsilon_{r}\right\}\left[E_{r}\right]\left\{\varepsilon_{r}\right\} r d A d \theta
$$

where $\quad \varepsilon_{r}=\left(r v,_{\theta}+r w+z v,_{\theta}-z w,,_{\theta}\right) / r^{2}$

and $A$ refers to the cross section of the ring. The first and second terms in Eq. (15) give the membrane strain in the ring and are seen to be the same as for the shell as given in Eq. (10). The third and fourth terms in Eq. (15) give the bending strain in the ring. In the present formulation, it is assumed that the cross section of each ring is constant around the circumference. Therefore, the properties of the ring cross section given by the 
integrals $\int_{A} z^{i} d A ; i=0,1,2$ that occur in Eq. (14) are not a function of $\theta$ and can be readily evaluated and summed for the rectangles used to define a ring cross section.

\section{Implementation of Method}

A goal in this development was to make the formulation of the new, design-oriented fuselage analysis method analogous to the formulation that was used in the equivalent plate analysis method for wings. This approach facilitates the implementation of the two analyses in a single version of the ELAPS code. In particular, procedures that were developed for plate analysis are also used in shell analysis. One example is the use of the special library of subroutines that exists in ELAPS to perform the operations of addition, subtraction, multiplication, differentiation and integration of the terms in the high-order, lengthy polynomial expressions that result when geometrical functions and assumed displacement functions are substituted into the energy equation. Use of these subroutines allow all operations to be performed in an exact, closed-form manner and result in a straightforward, simplified coding implementation of these lengthy, tedious polynomial expressions. However, in order to use these special subroutines, all expressions must be in polynomial form.

Unlike the strains for plates, the strains for shells and rings contain terms that cannot be expressed in standard polynomial form. Functions of the shell radius, $r$, that is expressed as a polynomial in Eq. (1), are contained in the denominator of expressions for the shell and ring strains, Eqs. (9), (10), (11) and (15). In addition, the quantity $s,_{x}$ in Eq. (13) is the square root of a polynomial that cannot be manipulated by the special set of subroutines. The terms $1 / r$ and $s,_{x}$ are both functions of the shell radius. In order to be able to utilize the advantages of the special subroutines, quantities containing these terms are approximated by standard polynomial functions. Each quantity containing these terms is evaluated at a set of points along the length of a fuselage segment and a leastsquares fit is made through these points to obtain a polynomial that approximates the original geometric quantity. The resulting polynomials can be combined with other polynomials in the strain energy equations using operators in the special subroutines. This procedure allows closed-form integration to be used to form sets of integral tables that are subsequently used to assemble the stiffness matrix for the structural model. This procedure has been demonstrated to be very computationally efficient in applications of the equivalent-plate analysis. This approximation approach was readily implemented in the ELAPS code and offered efficiency advantages over the conventional approach of using Gaussian integration that was used to implement similar ring-frame energy equations in Ref. 7.

\section{Connecting Structural Segments}

Spring elements are used to connect the structural segments that comprise an airframe model. Use of such springs to connect adjacent shell elements along their common circumferential boundaries is illustrated in Fig. 4. A typical connection is shown by the springs that are located along circumferential boundaries of the shell segment between the second and fourth rings in the illustrative figure. In addition, the springs that are shown located along the longitudinal boundary are used to impose appropriate boundary conditions at the plane of symmetry. Translational springs in the $x, y$, and $z$ directions and a rotational spring about the $x$-axis can be defined at each location indicated by the symbols. The rotational springs are used to connect the circumferential bending deformation that is governed by ring frame stiffnesses of adjacent shell segments. The strain energy of such connectors between segment $\mathrm{A}$ and segment $\mathrm{B}$ is given by

$$
\Omega_{\text {spring }}=1 / 2 k_{s}\left(\delta_{\text {segA }}-\delta_{\text {segB }}\right)^{2}
$$

where spring stiffnesses, $k_{s}$, are defined for each of the three translations and the rotation. In the displacement quantities, $\delta_{\text {segA }}$ and $\delta_{\text {segB }}$, the radial and circumferential displacements of a shell must be transformed to displacements in the $y$ and $z$ directions before calculation of the spring's contribution to the global stiffness matrix. When the springs are used to imposed boundary conditions on a model, the displacements for one of the segments is taken to have the value of zero. These connector springs are also used to connect adjacent plate segments by locating translations springs at the upper and lower surfaces of their common boundaries.

Finally, these connector springs are used to connect shell segments to plate segments. Typical shell-toplate connections are shown in Fig. 4. The connector springs do not have to be located at any particular points along the intersection of two segments, since both the model geometry and the assumed displacements are defined as continuous functions over the segments. Individual springs can be defined as well as a set of evenly-spaced springs along a segment edge. This 
method of connecting segments provides significant versatility for constructing models and facilitates making design changes such as shifting the longitudinal location of the wing/body intersection. Such definitions and modifications are time-consuming and difficult to automate when using conventional finiteelement methods.

\section{Applications and Results}

The fuselage structural analysis procedure will be applied to examples that illustrate various features of the new method. Two examples are presented, (1) a ring-stiffened cantilever cylinder that is used to assess the accuracy of the fuselage formulation and (2) a platering model that is used to demonstrate the connection of plate and ring segments.

\section{Cantilever Cylinder Model}

An example application of a ring-stiffened cantilever cylinder that has been studied in Refs. 8 and 9 is shown in Fig. 5. The cylinder has a length of 60.0 in., radius of $15.0 \mathrm{in}$. and a wall thickness of $0.032 \mathrm{in.}$ Four stiffening rings are evenly spaced along the length of the cylinder. These rings have a rectangular crosssection with a height of $0.78 \mathrm{in}$. and a width of $1.00 \mathrm{in}$. The material in both the cylinder wall and rings is aluminum with a Young's modulus of $E=10.6 \times 10^{6}$ psi and a Poisson's ratio of $v=0.33$. The cylinder is modeled with a single shell segment that spans the entire length. Only half of the cylinder is modeled since symmetry boundary conditions are applied by constraining deflections in the $y$-direction and rotations about the $x$-axis along shell edges in the $x-z$ plane, at $\theta$ $=-90^{\circ}$ and at $\theta=+90^{\circ}$. The four rings are modeled as discrete members by smearing their stiffness properties over $1.0 \mathrm{in}$. wide shell segments at each of the ring locations.

\section{Cantilever Cylinder Results}

A static analysis is performed with a radial point load of $1000 \mathrm{lb}$. in the positive $z$-direction at the free end. The deformed shapes of rings 1-3 from the ELAPS analysis are shown in Fig. 6. The displacement values were multiplied by a factor of 40 for illustrative purposes. The ring-stiffened cylinder was also analyzed using a conventional finite-element method ${ }^{10}$. In Table 1 , numerical values of radial displacements at $\theta=-90^{\circ}$, $0^{\circ}$, and $+90^{\circ}$ on the free end that are calculated using the ELAPS model are compared with results from the finite-element model (FEM). A maximum power of 8 was used on the $x$ and $\theta$ terms in the assumed displacement functions for $u, v$ and $w$ in the ELAPS analysis. Results are shown for three different levels of FEM modeling refinement. The entire circumference of the cylinder was represented in the FEM, not a symmetric half-model. The cylinder wall was modeled using a single row of finite elements between the rings. The least refined model, FEM 1, had 12 joints around the circumference and four constant strain triangles were used for each skin element as in Ref. 8. Linear strain quadrilateral elements were used to model the cylinder wall in FEM 2 and FEM 3 with the number of circumferential joints increased to 24 in FEM 3 . Agreement between the displacements from the most refined FEM model, FEM 3, and displacements from ELAPS is within 5 percent.

The rings in the finite-element models are modeled with straight beam elements between joints in the circumferential direction. A comparison of the moments in ring 1 from the ELAPS and FEM 3 analyses are shown in Fig. 7. Similar results for rings 2 and 3 are shown in Fig. 8. The curves for moments from ELAPS are in good agreement with the curves from the FEM analysis. The largest differences occur on the ELAPS segment boundaries at $\theta=-90^{\circ}$ and $\theta=$ $+90^{\circ}$. The difference at $\theta=-90^{\circ}$ on ring 2 is the largest. It appears that the displacement functions that span the entire half model used by ELAPS give a good representation of the overall moments but provide less accuracy in localized areas. Comparisons of the distribution of shear stresses in the cylinder wall from ELAPS and the FEM 3 analyses are shown in Figs. 9 and 10. These shear stresses are evaluated mid-way between rings 1 and 2 for Fig. 9 and mid-way between rings 2 and 3 in Fig. 10 . There is good overall agreement in the curves from both methods.

The comparisons of displacements, moments and shear stresses for the ring-stiffened cylinder indicate that the new design-oriented analysis method in ELAPS provides adequate accuracy for use during conceptual design.

\section{Plate-Ring Model}

The second example application is a plate-ring model shown in Fig. 11. A half-cylinder is connected to two plates to demonstrate the connection of plate and ring segments. This relatively simple example is representative of more general plate-shell connections since the bending of plate segments are reacted by the bending stiffness of the rings in a stiffened shell. The 
plates are connected to the ring by sets of connecting springs shown as the large, solid circular symbols. Clamped boundary conditions are imposed at the inner edge of the inboard plate. The thicknesses of the upper and lower skins of the plates and the inner and outer flanges of the ring have a constant value of 0.20 inches. The material properties of aluminum from the previous example are used for analysis purposes. The dimensions of this plate-ring structure are constant in the direction normal to the cross-section shown in Fig. 11. Therefore, only a strip of unit width is modeled.

Although the geometry of this model has been simplified for illustrative purposes, it is still somewhat representative of a semi-span wing model for a channelwing aircraft configuration. Channel-wing aircraft have been investigated because of their potential benefits for short take-off and landing operations ${ }^{11}$. An engine is mounted inside of the semi-circle. The high velocity of the airflow in the semi-circular region that is produced by the engine generates increased lift over that portion of the wing at low velocities of the aircraft.

\section{Plate-Ring Results}

A static analysis is performed with a 1.0 psi upward pressure load applied to the outboard plate segment for illustrative purposes. The vertical deflection of the plate-ring model is shown as a function of semispan location in Fig. 12. The deflection of the plate-ring model shown by the dashed curve is compared to the deflection of a cantilever plate model shown by the solid curve. The cantilever plate has an overall length of $250.0 \mathrm{in}$. and a constant depth of $10.0 \mathrm{in}$. The ELAPS model used to generate the solid curve was created by replacing the semi-circular ring segment with a 100.0 inch long plate segment. The deflection of the platering model is greater that the cantilever plate model because the flexibility of the $100.0 \mathrm{in}$. diameter ring segment is greater than that of a 100.0 in. long plate segment.

The tensile stress in the lower cover of the plate segments and outer flange of the ring segment is shown as a function of semispan location in Fig. 13. Again, the stresses for the plate-ring model are compared with stresses for the cantilever plate model. The stresses for both the inboard and the outboard plate segments are the same for both models since the bending moments from the applied loads are the same. However, the stresses in the outer flange of the ring differs from the stresses in the lower skin of the cantilever plate model. This difference is caused by the manner in which the pressure loading that is applied to the outer plate segment is carried by the ring segment and the corresponding plate segment in the cantilever plate model. Tensile stresses in lower surface of the cantilever plate model is the result of only the applied moment on the plate crosssection that is produced by the applied pressure loading. Stresses in the outer flange of the ring segment is the result of the applied moment and a vertical component of force. This vertical component of force increases the outer flange stress in the outer $90^{\circ}$ portion of the ring and decreases the stress in the inner $90^{\circ}$ portion.

\section{Concluding Remarks}

A design-oriented analysis capability for aircraft fuselage structures is described. The analytical model is defined using polynomial functions that minimize the amount of input data preparation and also facilitate any subsequent modifications that are made to the model during design. The analysis is based on the Ritz method and uses strain-energy equations from ring and shell theory. Example results are presented to indicate that the accuracy of this new structural analysis method is sufficient for use in conceptual design. The use of connector springs to couple plate segments and ring segments is demonstrated. The capability to couple ring-stiffened shell segments with plate segments within the ELAPS code provides a new design-oriented tool for modeling and analyzing entire airframes. 


\section{Appendix A \\ Shell Strain Coordinate Transformation}

The detailed steps of transforming the shell strains in shell coordinates of Eqs. (5)-(7) to strains in Cartesian coordinates are presented in this Appendix. First, the displacements $\bar{u}, \bar{v}$ and $\bar{w}$ in shell coordinates and the displacements $u, v$, and $w$ in Cartesian coordinates are considered. The circumferential displacements are the same in both coordinate systems, i.e., $\bar{v}=v$. The remaining two sets of displacements are shown in the following sketch.

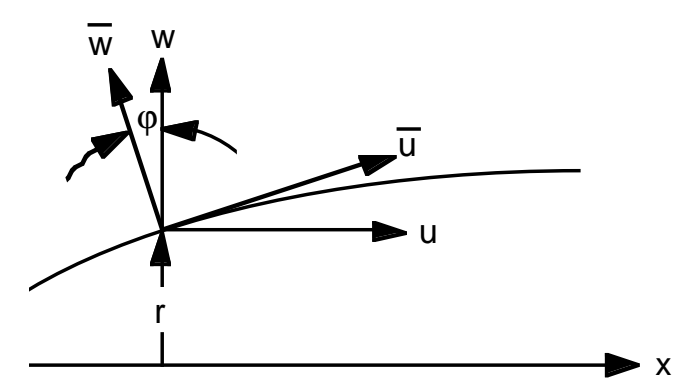

The transformation equations between the two sets of displacements are

$$
\left\{\begin{array}{l}
\bar{u} \\
\bar{w}
\end{array}\right\}=\left[\begin{array}{cc}
\cos \varphi & \sin \varphi \\
-\sin \varphi & \cos \varphi
\end{array}\right]\left\{\begin{array}{l}
u \\
w
\end{array}\right\}
$$

The trigonometric terms in Eq. (A.1) can be converted to differential form using the quantities shown in the next sketch

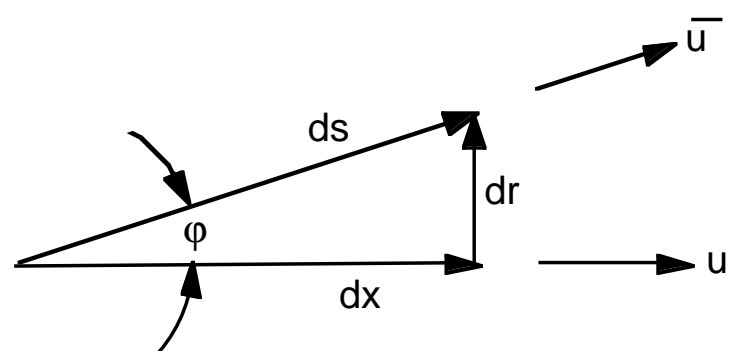

with $\quad \cos \varphi=\mathrm{d} x / \mathrm{d} s=1 / \mathrm{s}_{\mathrm{x}}$ and $\quad \sin \varphi=\mathrm{d} r / \mathrm{d} s=(\mathrm{d} r / \mathrm{d} x) /(\mathrm{d} s / \mathrm{d} x)=r_{x} / s_{,}$.

Substituting Eqs. (A.1) in differential form into Eqs. (5)-(7) gives $\varepsilon_{s}=\left(x,,_{s s}-\frac{r, r_{s}}{R_{1}}\right) u+x,,_{s} u,,_{s}+\left(r,{ }_{s s}+\frac{x, s_{s}}{R_{1}}\right) w+r,,_{s} w$,

$\varepsilon_{\theta}=\left(\frac{x,,_{s} r,_{s}}{r}-\frac{r, s_{s}}{R_{2}}\right) u+\frac{v,_{\theta}}{r}+\left(\frac{r,{ }_{s}^{2}}{r}+\frac{x,,_{s}}{R_{2}}\right) w$

$\varepsilon_{s \theta}=\left(x,{ }_{s} u,{ }_{\theta}-r,{ }_{s} v+r v,{ }_{s}+r,{ }_{s} w,{ }_{\theta}\right) / r$

Now, the derivatives of quantities, [ ], with respect to $s$ are expressed as derivatives with respect to $x$.

[]$_{s}=\frac{d[]}{d s}=\frac{d[]}{d x} \frac{d x}{d s}=[],{ }_{x} / s,{ }_{x}$

and

[]$_{s s}=\frac{d[]_{s}}{d x} \frac{d x}{d s}=[]_{x x} s,_{x}^{-2}-[]_{x} s,,_{x}^{-3} s,_{x x}$

Next, the differential quantities in shell coordinates, $s,_{x}$ and $s,_{x x}$, are converted to differentials quantities in the radial coordinate, $r_{x}$ and $r{ }_{x x}$, using the differential relation from consideration of the preceding sketch

$$
\begin{aligned}
& d s^{2}=d x^{2}+d r^{2} \\
& \text { or } \quad s,{ }_{x}^{2}=1+r,_{x}^{2} \\
& \text { then } \quad s,{ }_{x}=\sqrt{1+r,{ }_{x}^{2}} \\
& \text { and } \quad s,{ }_{x x}=r,{ }_{x} r,{ }_{x x} / \sqrt{1+r,{ }_{x}}
\end{aligned}
$$

The shell curvatures, $1 / R_{1}$ and $1 / R_{2}$, in shell coordinates can be expressed in Cartesian coordinates as

$$
\begin{aligned}
\frac{1}{R_{1}} & =\frac{-r, x_{x x}}{\left(1+r,_{x}^{2}\right)^{3 / 2}} \\
\text { and } \quad \frac{1}{R_{2}} & =\frac{\cos \varphi}{r}=\frac{1}{r s,,_{x}}
\end{aligned}
$$

Substitution of Eqs. (A.5)-(A.12) into Eqs. (A.2)-(A.4) gives the strain equations in Cartesian coordinates as shown in Eqs. (9)-(11). Note that values of zeros are obtained when the coefficients for the $u$ and $w$ displacements in Eq. (A.2) are evaluated. Similarly, the coefficient of the $u$ displacement in Eq. (A.3) is found to be equal to zero. 


\section{$\underline{\text { References }}$}

1 Giles, G.L.: Equivalent Plate Analysis of Aircraft Wing Box Structures with General Planform Geometry. J. of Aircraft, Vol. 23, No. 11, November 1986, pp. 859-864.

2 Giles, G.L.: Further Generalization of an Equivalent Plate Representation for Aircraft Structural Analysis. J. of Aircraft, Vol. 26, No. 1, January 1989, pp. 67-74.

3 Livne, E.; Sels, R.A.; and Bhatia, K.G.: Lessons from Application of Equivalent Plate Structural Modeling to an HSCT Wing. J. of Aircraft, Vol. 31, No. 4, July-Aug. 1994, pp. 953-960.

${ }^{4}$ Livne, E.: Equivalent Plate Structural Modeling for Wing Shape Optimization Including Transverse Shear. AIAA Journal, Vol. 32, No. 6, June 1994, pp.12781288.

5 Giles, G.L.: Equivalent Plate Modeling for Conceptual Design of Aircraft Wing Structures. AIAA Paper No. 95-3945, 1st AIAA Aircraft Engineering, Technology and Operations Congress, Los Angeles, CA, Sept. 19-21, 1995.
6 Bushnell, D.: Computerized Analysis of Shells Governing Equations. Computers \& Structures, Pergamon Press Ltd., Vol. 18, No. 3, 1984, pp. 471536.

7 Bowman, L.M.: Analysis of Fuselage Frames with General Circumferential Shape using the Ritz Method. George Washington University Master's Thesis, July 1991.

8 Turner, M.J.; Martin, H.C.; and Weikel, R.C.: Further Development and Applications of the Stiffness Method. In Matrix Methods of Structural Analysis, Pergamon Press, 1964, pp.204-266.

9 Langelaan, J.W.; and Livne, E.: Analytical Sensitivities and Design Oriented Structural Analysis for Airplane Fuselage Shape Synthesis. Computers \& Structures, Pergamon Press Ltd., Vol. 62, No. 3, 1997, pp. 505-519.

10 Whetstone, W.D.: EISI-EAL Engineering Analysis Language Reference Manual--EISI-EAL System Level 2091, Engineering Information Systems, Inc., July 1983.

11 Blick, E.F.; and Homer, V.: Power-on Channel Wing Aerodynamics. J. of Aircraft, Vol. 8, No. 4, April 1971, pp. 234-238.

Table 1. Comparison of displacements

\begin{tabular}{llll}
\hline \multirow{2}{*}{ Model } & \multicolumn{3}{c}{ Radial displacement of free end } \\
\cline { 2 - 4 } & $\theta=-90^{\circ}$ & $\theta=0^{\circ}$ & $\theta=90^{\circ}$ \\
\hline ELAPS $^{\mathrm{a}}$ & -0.2056 & 0.1177 & -0.0671 \\
FEM 1 $^{\mathrm{b}}$ & -0.1903 & 0.1030 & -0.0601 \\
FEM 2 $^{\mathrm{c}}$ & -0.1982 & 0.1086 & -0.0644 \\
FEM 3 $^{\mathrm{d}}$ & -0.2037 & 0.1098 & -0.0660 \\
\hline
\end{tabular}

${ }^{\text {a }}$ The ELAPS model uses maximum power of 8 on $x$ and $\theta$ in the displacement functions.

${ }^{\mathrm{b}}$ FEM 1 has 12 joints per ring and four constant strain triangles are used for each skin element.

${ }^{c}$ FEM 2 has 12 joints per ring and linear strain quadrilateral elements are used for the skin.

${ }^{d}$ FEM 3 has 24 joints per ring and linear strain quadrilateral elements are used for the skin. 


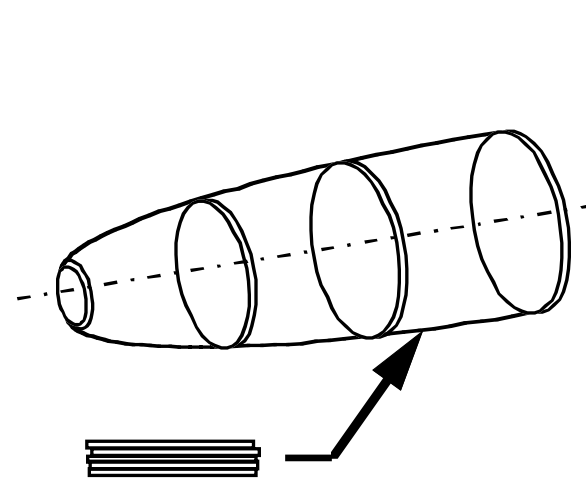

Membrane shell with layered composite skin.

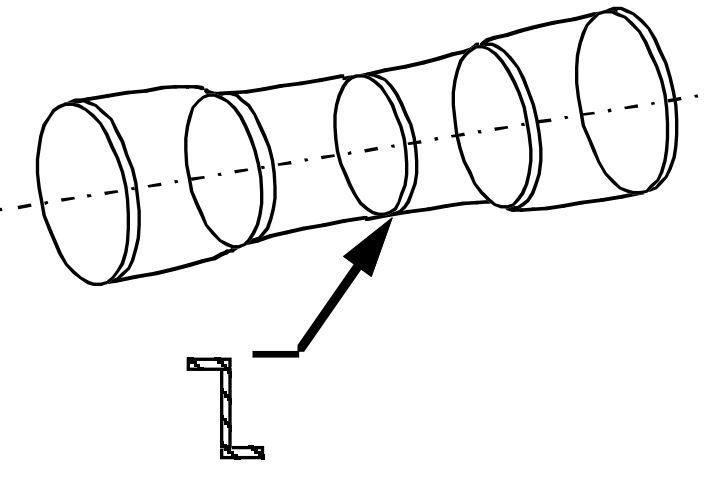

Frame cross-section defined using three rectangles.

Fig. 1 Analytical modeling of fuselage structure.

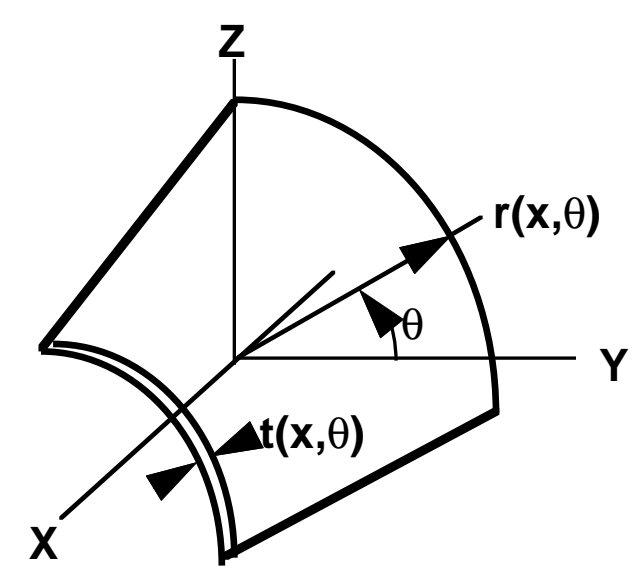

(a) Geometry definition

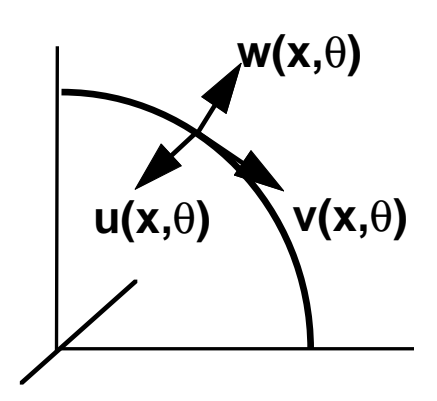

(b) Displacement functions

Fig. 2 Shell segment definition. 


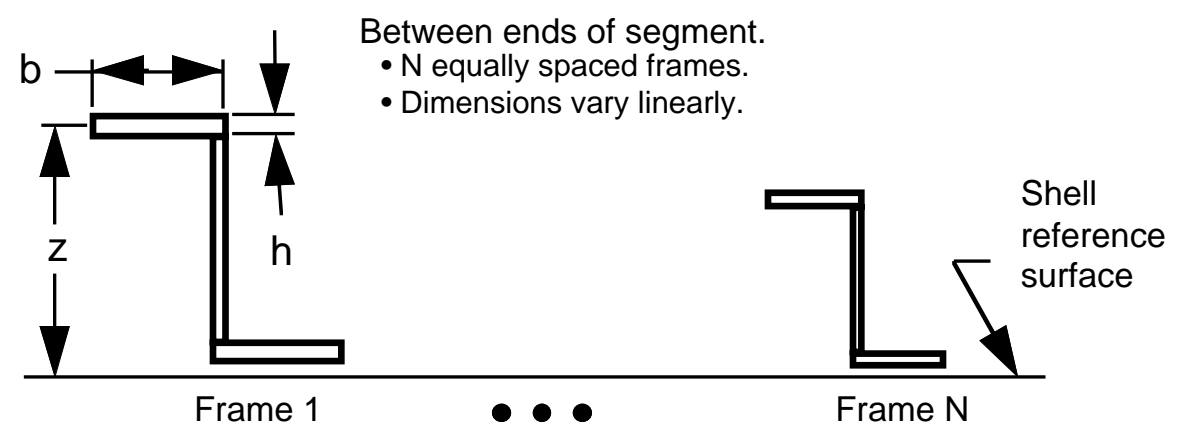

Fig. 3 Fuselage frame definition.

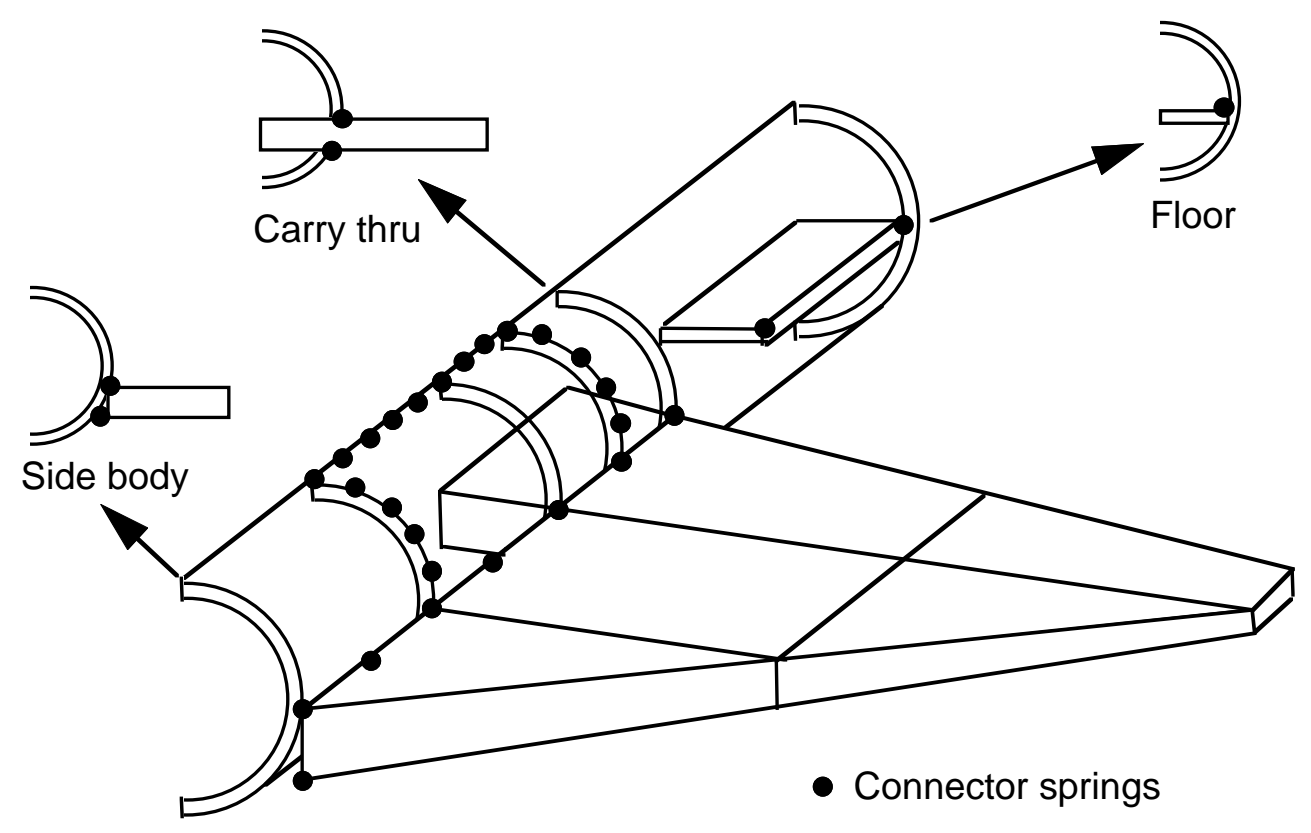

Fig. 4 Coupling of segments using connector springs. 


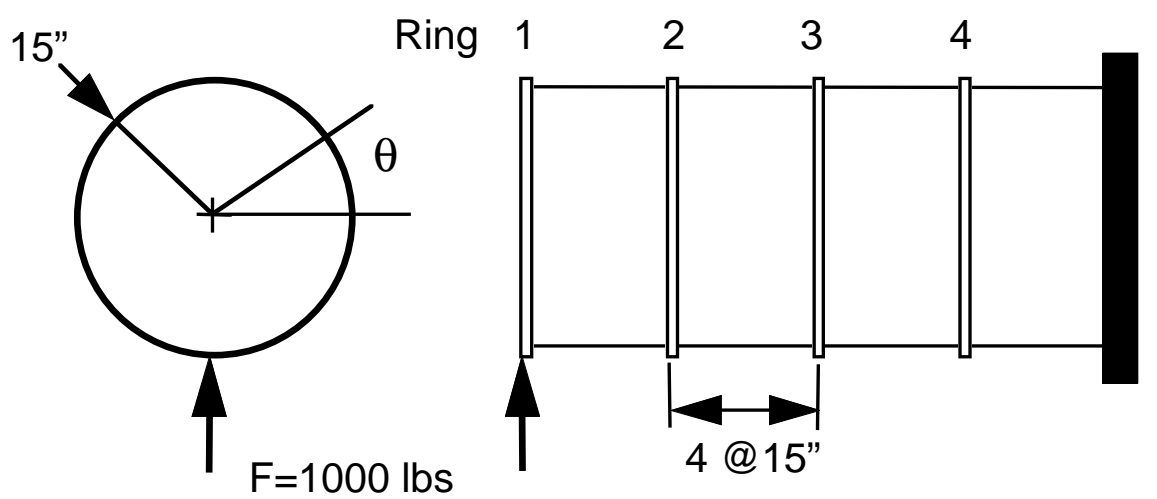

Fig. 5 Cantilevered ring-stiffened cylinder.

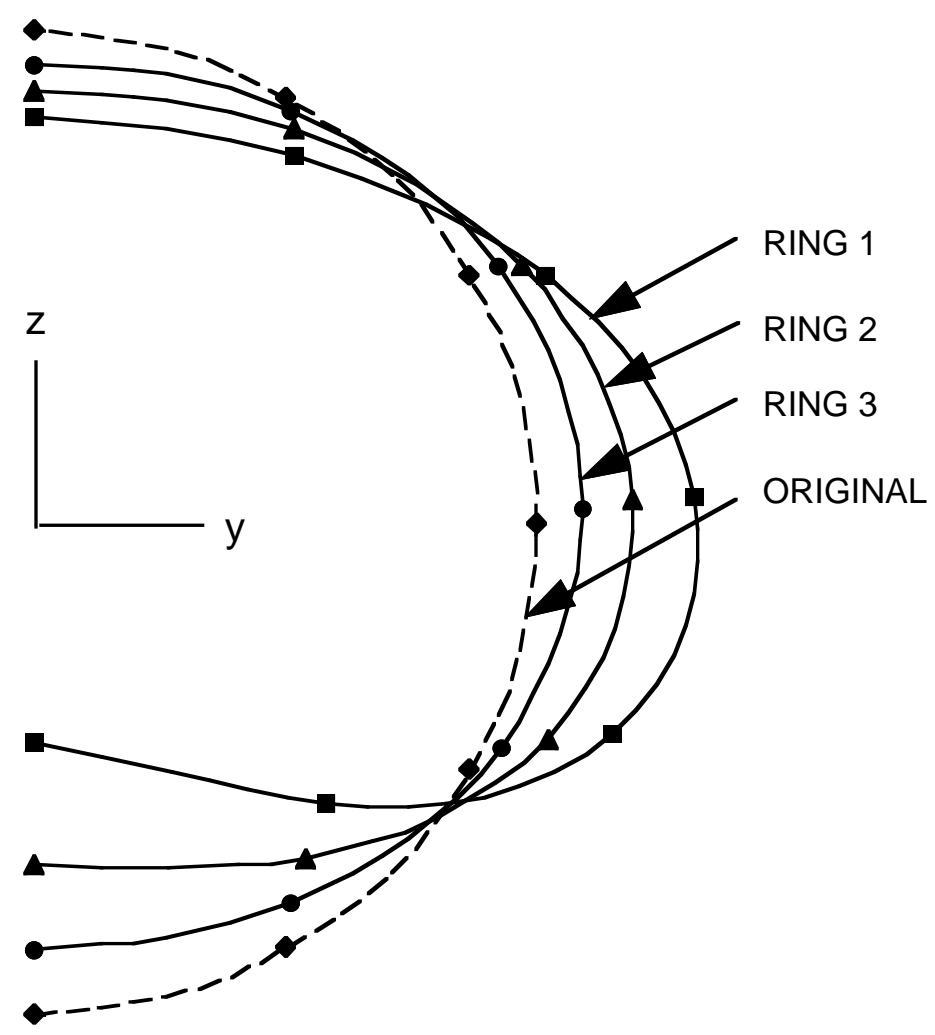

Fig. 6 Ring displacements. 
AIAA-98-1749

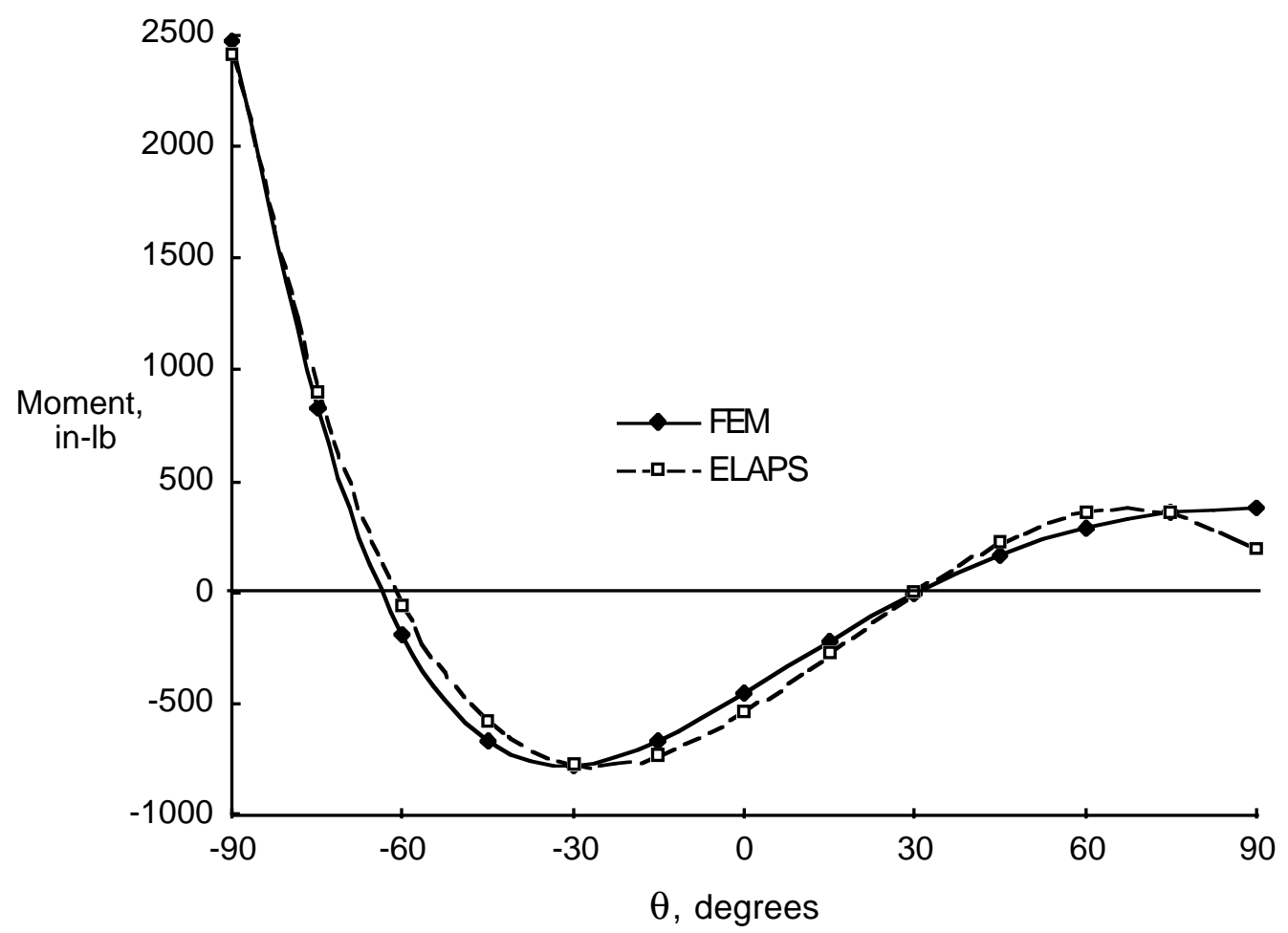

Fig. 7 Moments in ring 1.

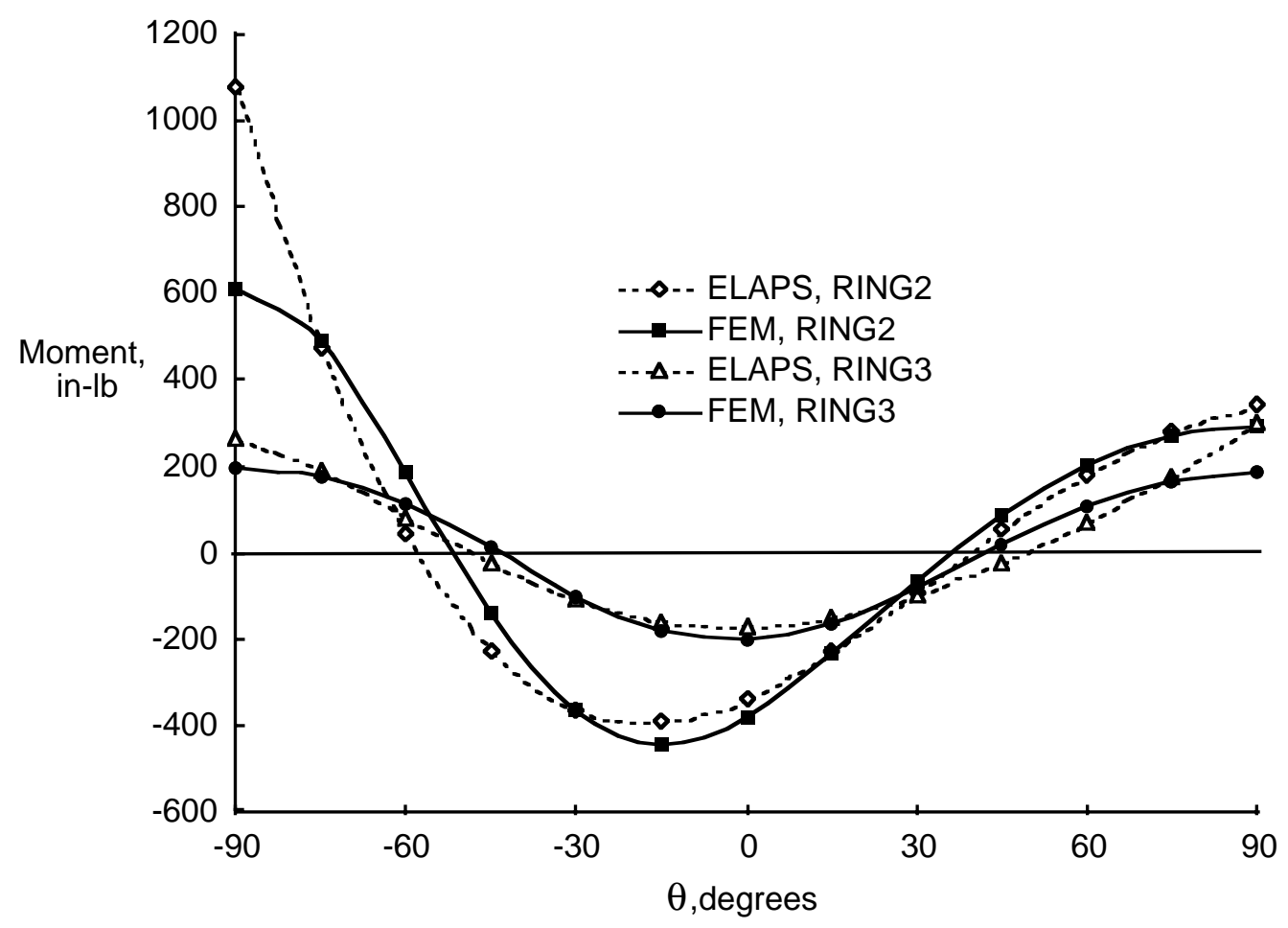

Fig. 8 Moments in ring 2 and ring 3. 
AIAA-98-1749

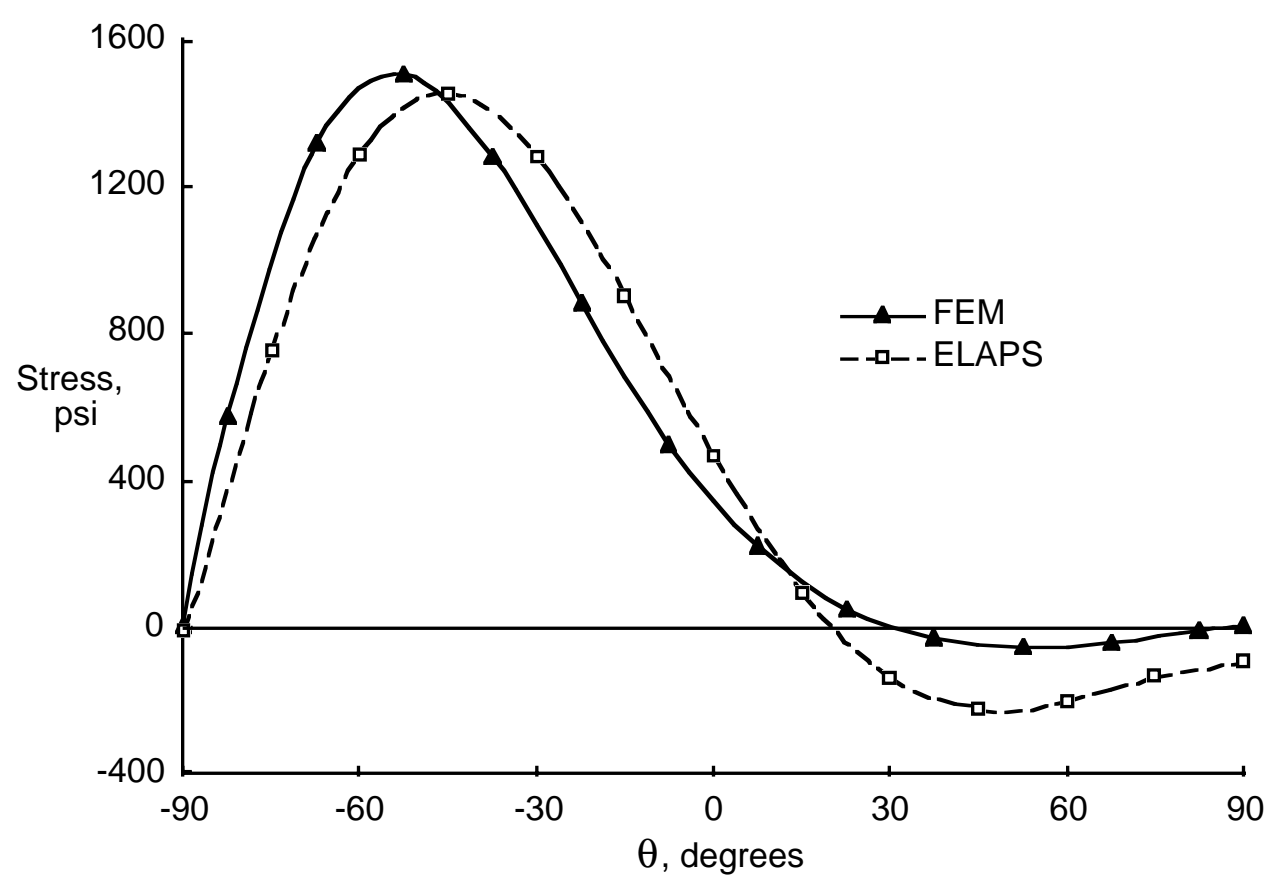

Fig. 9 Shear stress between ring 1 and ring 2.

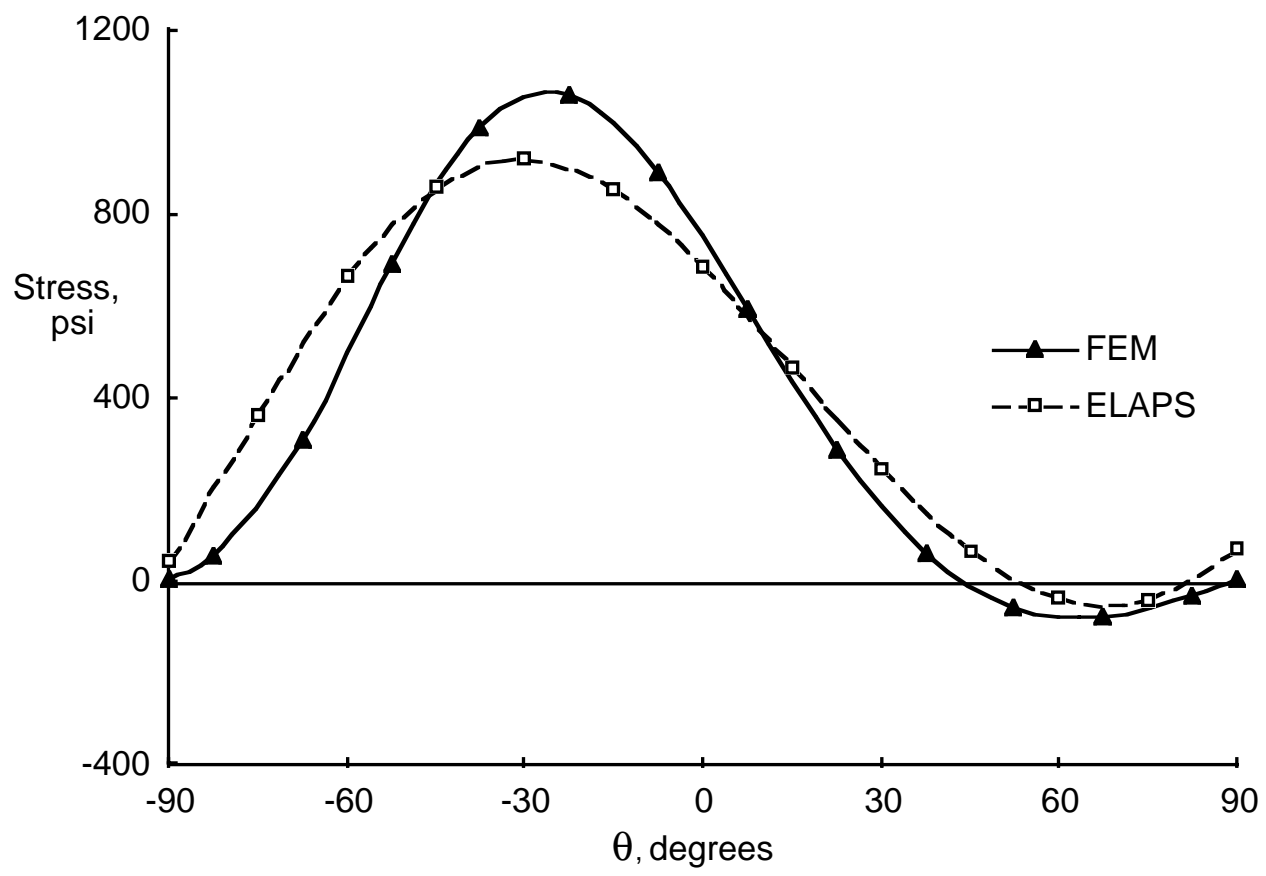

Fig. 10 Shear stress between ring 2 and ring 3. 
AIAA-98-1749

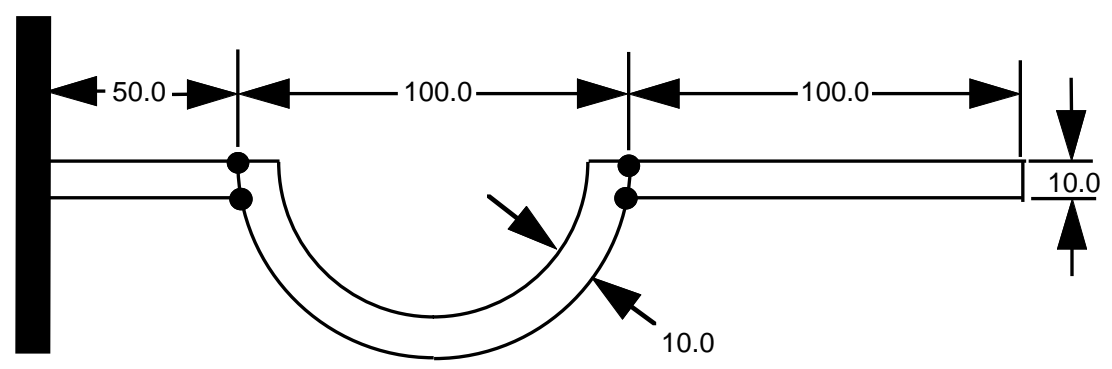

Fig. 11 Plate-ring model of channel wing structure.

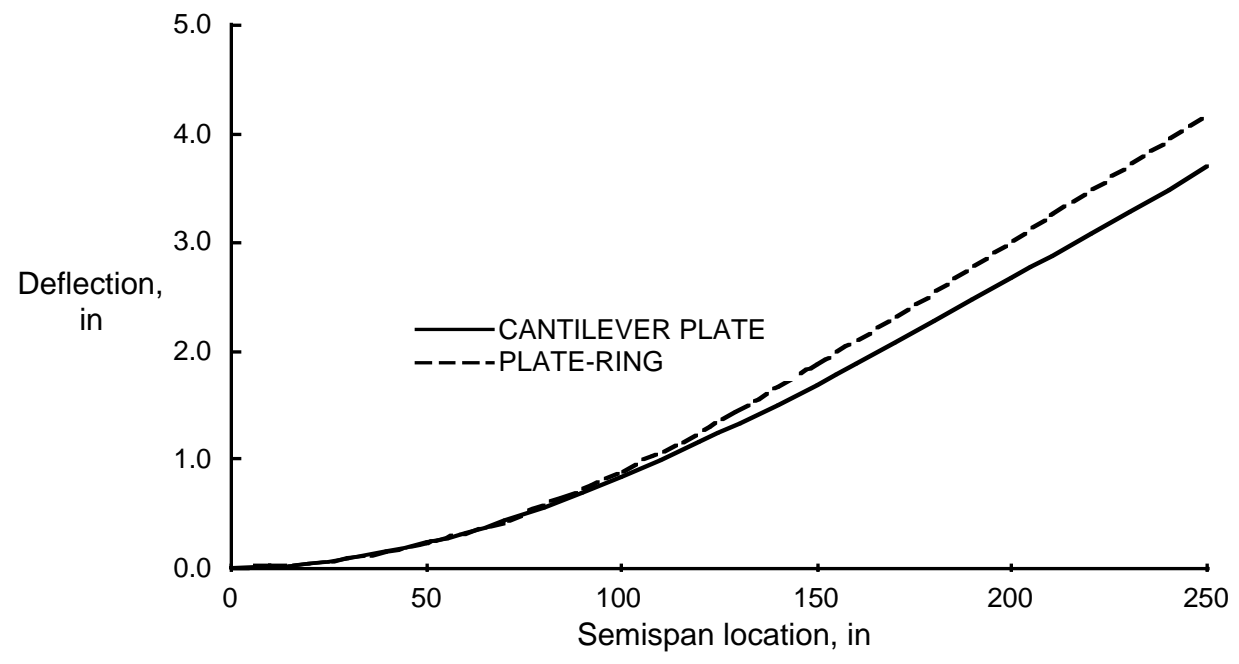

Fig. 12 Vertical deflection of wing structures.

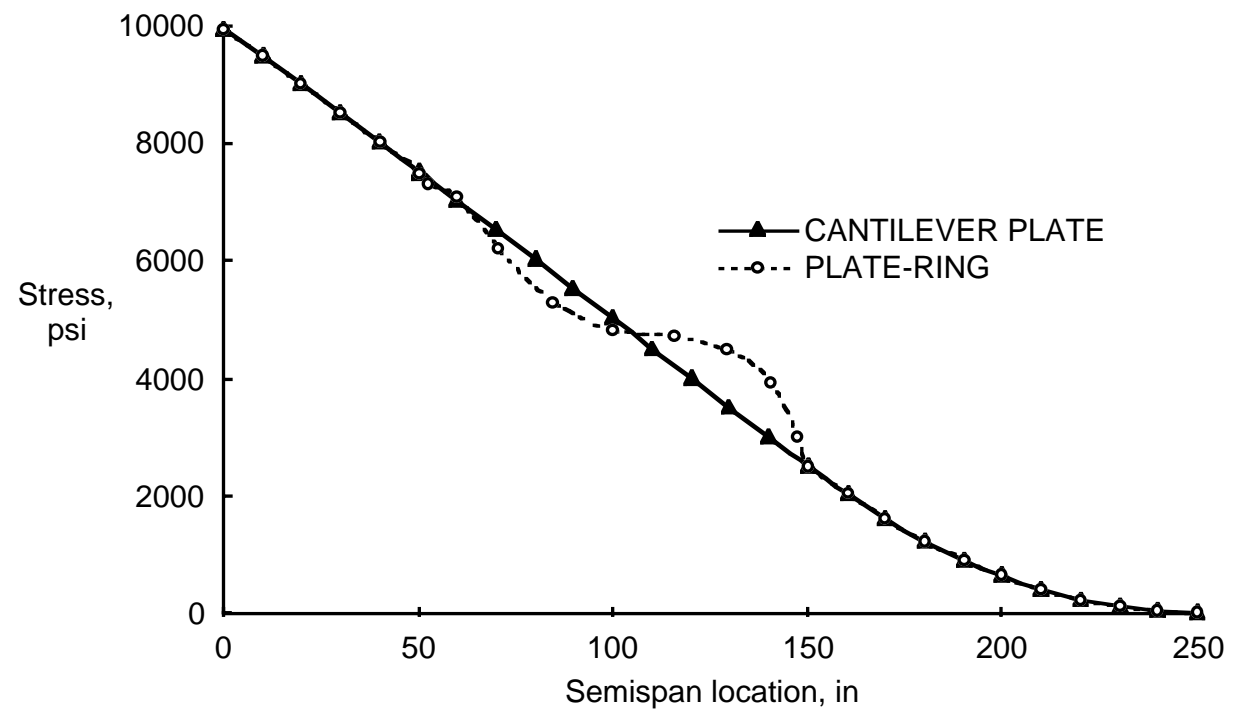

Fig. 13 Stress in lower cover skin. 